\title{
Dose influence on the PMMA e-resist for the development of high-aspect ratio and reproducible sub-micrometric structures by electron beam lithography
}

A. Veroli, F. Mura, M. Balucani, and R. Caminiti

Citation: AIP Conference Proceedings 1749, 020010 (2016); doi: 10.1063/1.4954493

View online: https://doi.org/10.1063/1.4954493

View Table of Contents: http://aip.scitation.org/toc/apc/1749/1

Published by the American Institute of Physics

\section{Articles you may be interested in}

Optimization of an electron beam lithography instrument for fast, large area writing at $10 \mathrm{kV}$ acceleration voltage Journal of Vacuum Science \& Technology B, Nanotechnology and Microelectronics: Materials, Processing, Measurement, and Phenomena 31, 043202 (2013); 10.1116/1.4813325

Sub-10 nm electron beam lithography using cold development of poly(methylmethacrylate)

Journal of Vacuum Science \& Technology B: Microelectronics and Nanometer Structures Processing, Measurement, and Phenomena 22, 1711 (2004); 10.1116/1.1763897

Influence of electron-beam lithography exposure current level on the transport characteristics of graphene field effect transistors

Journal of Applied Physics 119, 124502 (2016); 10.1063/1.4944599

Comparison between ZEP and PMMA resists for nanoscale electron beam lithography experimentally and by numerical modeling

Journal of Vacuum Science \& Technology B, Nanotechnology and Microelectronics: Materials, Processing, Measurement, and Phenomena 29, 06F306 (2011); 10.1116/1.3640794

Electron beam lithography using a PMMA/P(MMA 8.5 MAA) bilayer for negative tone lift-off process Journal of Vacuum Science \& Technology B, Nanotechnology and Microelectronics: Materials, Processing, Measurement, and Phenomena 33, 061602 (2015); 10.1116/1.4935129

Sub-10-nm half-pitch electron-beam lithography by using poly(methyl methacrylate) as a negative resist Journal of Vacuum Science \& Technology B, Nanotechnology and Microelectronics: Materials, Processing, Measurement, and Phenomena 28, C6C58 (2010); 10.1116/1.3501353 


\title{
Dose influence on the PMMA e-Resist for the development of high-aspect ratio and reproducible sub-micrometric structures by Electron Beam Lithography.
}

\author{
A. Veroli ${ }^{1,2}$, F. Mura ${ }^{3,4, a)}$, M. Balucani ${ }^{1,3}$ and R. Caminiti ${ }^{3,4}$ \\ ${ }^{1}$ Department of Information Engineering, Electronics and Telecommunications (DIET), University of Rome La \\ Sapienza, Rome, Italy \\ ${ }^{2}$ Department of Basic and Applied Sciences for Engineering (BASE), University of Rome La Sapienza, Rome, Italy \\ ${ }^{3}$ Research Center For Nanotechnology Applied To The Engineering (CNIS), University of Rome La Sapienza, Rome, \\ Italy \\ ${ }^{4}$ Department Of Chemistry, University of Rome La Sapienza, Rome, Italy \\ a) Corresponding author: francesco.mura@uniroma1.it
}

\begin{abstract}
In this work, a statistical process control method is presented showing the accuracy and the reliability obtained with of PMMA E-resist AR-P 672, using an Elphy Quantum Electron Beam Lithography module integrated on a FE-SEM Zeiss Auriga instrument. Reproducible nanostructures with an high aspect ratio between e-resist thickness and width of written geometric structure are shown. Detailed investigation of geometry features are investigated with dimension in the range of $200 \mathrm{~nm}$ to $1 \mathrm{~m}$. The adopted method will show how tuning the Area Dose factor and the PMMA thickness it was possible to determine the correct and reproducible parameters that allows to obtain well defined electron-beam features with a 4:1 aspect ratio. Such high aspect ratio opens the possibility to realize an electron-beam lithography lift-off process by using a standard e-beam resist.
\end{abstract}

\section{INTRODUCTION}

The dramatic development of the nanotechnologies during the last ten years has requested rapid advances in the nanofabrication for the submicron regime, and especially for the so-called top-down technique, where the pattern transfer happens at the nanometer scale (e.g. $100 \mathrm{~nm}$ ). Electron Beam Lithography (EBL) results to be one of the most used technique for obtaining nanostructures down to sub $10 \mathrm{~nm}$, using different strategies such e-resist development processed with cold developers $\left(4-10^{\circ} \mathrm{C}\right)$ or through a double layer resist system consisted in a $15 \mathrm{~nm}$ thick Chromium mask $[1,2,3]$.

Nevertheless, it exists also other different techniques for reaching this aim, as, for example, the immersion lithography, able to increase the resolution of classical deep UV lithography down to $20 \mathrm{~nm}$ using excimer lasers with wavelengths of 193 and $248 \mathrm{~nm}$ [4, 5], the nanoimprinting lithography (NIL) that creates patterns by mechanical deformation of imprint resist and subsequent processes [6, 7, 8], or the Focused Ion Beam (FIB) Lithography [9, 10].

However, the Electron Beam Lithography (EBL) mantains yet its leading role, because of its possibility to exist as stand-alone devices or integrated in a normal electron microscope, equipped with a beam blanker [11, 12]. Besides, EBL allows to obtain microsystems or nanostructures with high resolution, high reliability in processing, high accuracy in positioning/alignment, and high flexibility in pattern replication [13, 14]. These features are such that EBL has been used in different research areas like metamaterials and nanophotonic structures $[4,8,15,16]$, microfluidic devices [17, 18] and even in Bioscience and Medical field [19, 20].

Generally, in nano-fabrication field, the EBL plays a fundamental part in the Lift-Off technique, together with the use of the Poly-Methyl-Methacrylate (PMMA), or with the assistance of other materials [21, 22, 23]. The PMMA is the most common positive e-resist used for the EBL, and this is due for its flexibility and other well known features [11], as well for its possibility to reach high resolution [24, 25, 26]. As rule of thumb, the choice of the resist thickness should be done considering that the final aspect ratio of the wanted nanostructure should be less than three times the 
thickness of material to be deposited on the substrate[12, 27]. So, the main goal is to push the working limits of thick PMMA towards high aspect ratio, in order to have just a single layer of positive resist for different application in micro and nano-fabrication field, like, for example, the use of a single PMMA layer as sacrifical mask in a Lift-Off technique. In this paper we present a Statistic Process Control on $1.6 \mu \mathrm{m}$ thick PMMA AR-P 672 (Allresist, Germany), performed with an Raith Elphy Quantum EBL module, inspecting the quantity of electric charge per squared centimeters used to expose the e-resist, normally defined as Area Dose, for the formation of curved lines with different dimensions. At the end of this study we have obtained an aspect ratio of $4: 1$ between e-resist thickness and the CAD structures dimensions with an Area Dose windows range comprised between 500 and $1050 \mu \mathrm{C} / \mathrm{cm}^{2}$.

\section{MATERIAL \& METHODS}

The substrate used for this paper has been a standard electronic grade wafer silicon $<100>$ from (MEMC Electronic Material Spa) while the e-resist is the PMMA AR-P 672.08 (All-Resist-de, Germany). The e-resist is deposited by Spin-Coating WS-650-23B (Laurell Technologies Corporation), with the following operating conditions: $300 \mathrm{rpm}$ for $5 \mathrm{~s}$, followed by $2000 \mathrm{rpm}$ for $45 \mathrm{~s}$ and hot plate baking at $170^{\circ} \mathrm{C}$ for 5 minutes; the final thickness obtained is $1.6 \mu \mathrm{m}$. The PMMA has been characterized using the Atomic Force Microscopy (Icon, Veeco), whose results are reported in Figure 1.

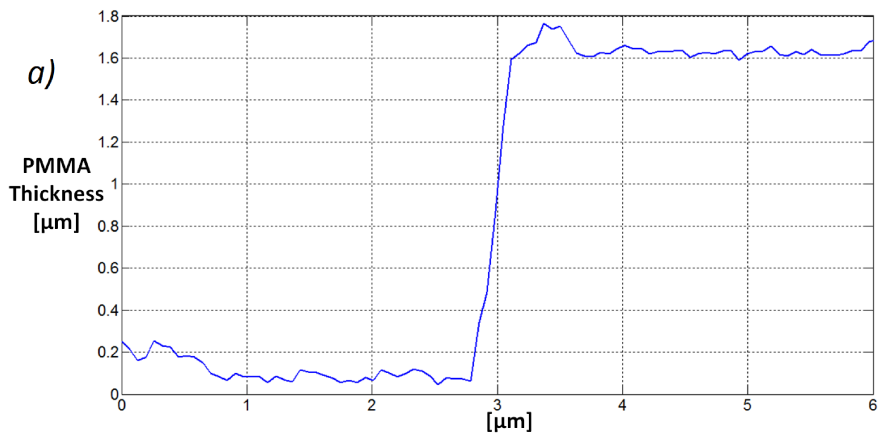

b)

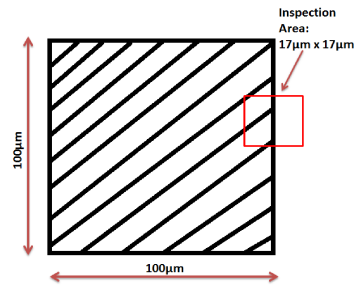

c)

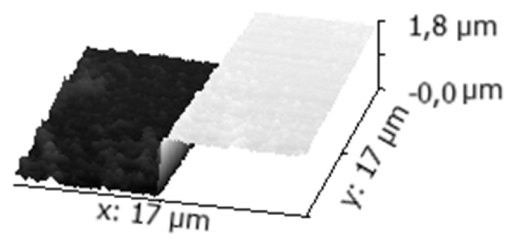

FIGURE 1. a) Line profile of the PMMA thickness obtained by Atomic Force Microscopy (AFM); b) dimensions of the inspected area; c) 3D image of the thickness variation inside the area of $17 \mu \mathrm{m} \times 17 \mu \mathrm{m}$ chosen for the measurement.

The Electron Beam Lithography has been performed using an Elphy Quantum EBL module, integrated in a FESEM Zeiss Auriga instrument, writing in longitudinal area mode. The patterned structure has been obtained through a CAD consisting of curved lines with different width and has been made with Raith GDSII v1.1 and reported in figure 2. The choice of this kind of geometry has been made considering the need to study the performance of the instrument on both linear and curvilinear elements, and to examine the interaction of the elements at different distances. The development phase has been performed using a Methyl Isobutyl Ketone (MIBK) solution diluted with Isopropyl Alcohol (IPA) in relation 1:3 for $75 \mathrm{~s}$ and then placed in a pure IPA solution for $30 \mathrm{~s}$. The patterned wafers has been analyzed with the Electron Microscopy. In order to prevent a deep variation on the morphology, caused by a direct contact between the unexposed e-resist and the electron beam, all the exposed and developed sample have been coated with a $20 \mathrm{~nm}$ Chromium layer using a metal sputter (Quorum Q150T). Then, all the images have been analyzed using ImageJ software to build up a Statistic Control with numerical methods in Matlab enviroment, so to obtain a 
direct correlation between the effective dimension of the printed structures with the area dose value set of the EBL instrument.

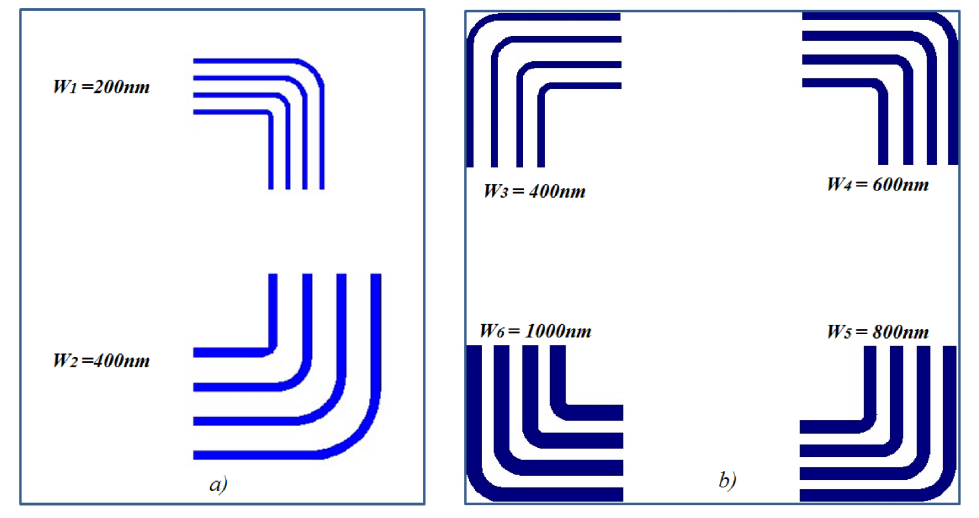

FIGURE 2. a) CAD used for the to the first writing cycle, having structures with width of $200 \mathrm{~nm}$ (W1) and $400 \mathrm{~nm}$ (W2); b) CAD used during the second writing cycle with width that changes from 400 to $1000 \mathrm{~nm}$. The width and the curvature radius of the structures showed in figure have the same dimensions.

\section{RESULT \& DISCUSSION}

In order to obtain a repeatable process window for an EBL writing process, a first writing session has been performed, taking as reference structure, the one showed in figure $1 \mathrm{a}$, and analyzing a wide range of dose, repeated with a 50 increasing dose steps. The performing parameters are reported in Tab. 1, where:

- The working distance is the distance between the sample and the electronic gun.

- The dwell time is the time that electronic beam remains fixed in a single point.

- The step size is the distance along the $\mathrm{x}$ axis between two points written by the EBL.

- The line spacing is the distance along the y axis between two points written by the EBL.

- The area dose is the quantity of electric charge per squared centimeters usedto expose the e-resist.

TABLE 1. :EBL parameter for the writings cycle.

\begin{tabular}{lcc}
\hline & First Writing Cycle & Second Writing Cycle \\
\hline Acceleration Voltage & $30 \mathrm{keV}$ & $30 \mathrm{keV}$ \\
Working Distance & $6 \mathrm{~mm}$ & $6 \mathrm{~mm}$ \\
Beam Current & $71.4 \mathrm{pA}$ & $85.4 \mathrm{pA}$ \\
Dwell Time & $0.002 \mathrm{~ms}$ & $0.0033 \mathrm{~ms}$ \\
Step Size & $6 \mathrm{~nm}$ & $6 \mathrm{~nm}$ \\
Line Spacing & $6 \mathrm{~nm}$ & $6 \mathrm{~nm}$ \\
Area Dose, start value & $350 \mu \mathrm{C} / \mathrm{cm}^{2}$ & $400 \mu \mathrm{C} / \mathrm{cm}^{2}$ \\
Area Dose, final value & $3350 \mu \mathrm{C} / \mathrm{cm}^{2}$ & $1500 \mu \mathrm{C} / \mathrm{cm}^{2}$ \\
Dose Steps & 50 & 25 \\
\hline
\end{tabular}

The SEM analysis of this first writing cycle has permitted to have a first clue value about the dose range and its repeatability. In fact, from the image analysis it has been possible to calculate the value of Normalization Deviation Standard (N-SD) for the obtained width from each group of curves (Eq. 1), that provides an information about the accuracy of the writing cycle respect the desired feature. Its known that values lower than 5\%, indicates an excellent repeatability [28]. 


$$
N-S D=\frac{\sqrt{\text { variance }}}{\text { mean value }}
$$

Observing the graph showed in Fig. 3, and highlighted by the regression line, it's evident that we have an increase of the structure dimensions proportional with the area dose value, where the determination value is $\mathrm{R}^{2}=0.9311$.

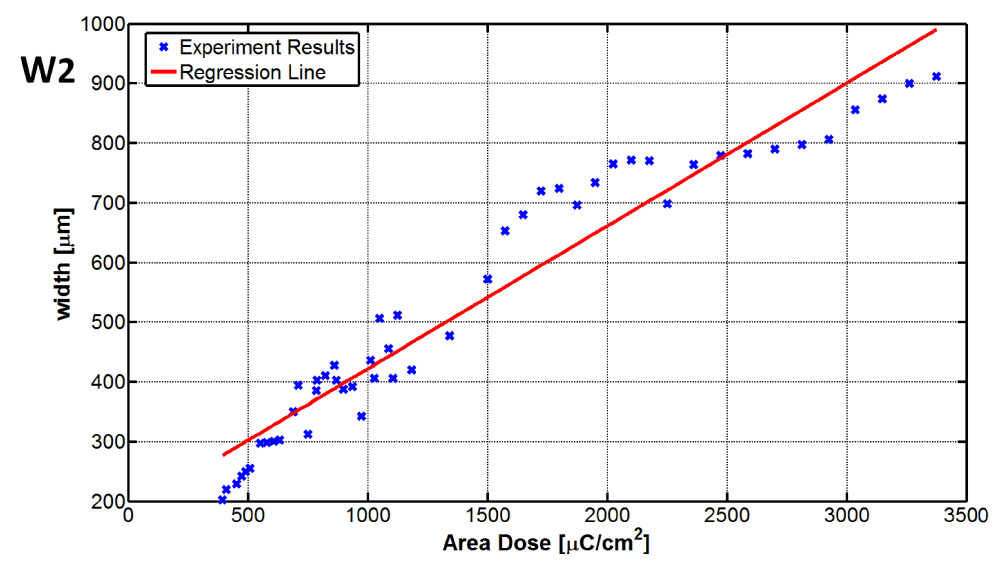

FIGURE 3. EBL first writing cycle result, on the $x$-axis are report the Area Dose value and on the y-axis the curved lines width.

Beside, from this data, it was obtained an important information about the Area Dose range (ADr) for the structures with thickness of 200 and $400 \mathrm{~nm}$. Broadening the graph around the area containing the points near the desired thickness (Figure 4), is possible to limit the ADr with high N-SD value. These data have been calculated from the graphical analysis of the SEM images of the first writing cycle. The red line represents the expected dimension, while the green one is the mean value obtained from the imaging analysis and finally, the two dashed lines correspond to the $\pm 3 \sigma$ error.

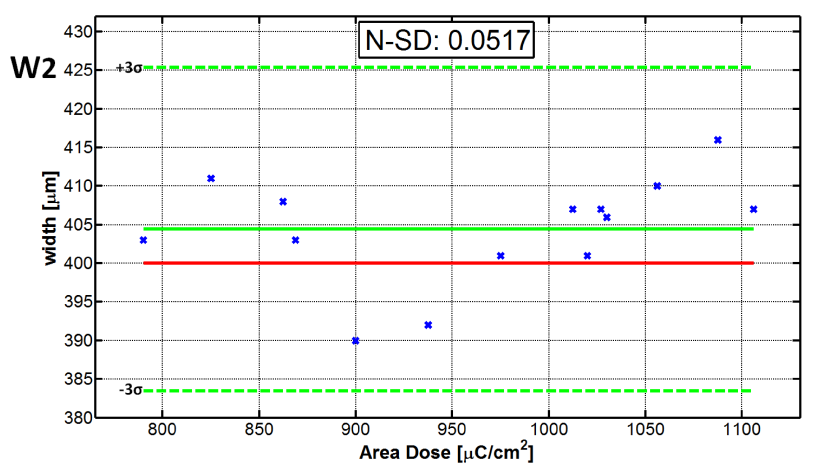

FIGURE 4. In the graph are reported all the experimental values of the $400 \mathrm{~nm}$ width structures (x) versus the Area Dose.

The results are that the $\operatorname{ADr} 869 \mu \mathrm{C} / \mathrm{cm}^{2}-1106 \mu \mathrm{C} / \mathrm{cm}^{2}$, for the structure with the width of 200 nm, showed a $\mathrm{N}-\mathrm{SD}$ of $12 \%$, while for the other structures with a width of $400 \mathrm{~nm}$, the ADr is $785.5 \mu \mathrm{C} / \mathrm{cm}^{2}-1185 \mu \mathrm{C} / \mathrm{cm}^{2}$, with an excellent N-SD of $5.1 \%$. The good quality of the patterning can be observed from Fig. 1a, where its reported the AFM profile of a EBL writing. In fact, considering also the effect due to the shape of the AFM cantilever, it can be seen that the polymer wall created after the lithography has an angle close to the $88^{\circ}$ degree. For this reason, is possible to claim that for the $400 \mathrm{~nm}$ structures, we have performed a good lift off process of 4:1 aspect ratio. Besides, the high $\mathrm{N}-\mathrm{SD}$ value given by the $200 \mathrm{~nm}$ patterning, means that there are other aspects of the process, like the resist spinning, developing time and temperature, ecc. [29], that have to be carefully optimized in order to obtain more high aspect ratio. 

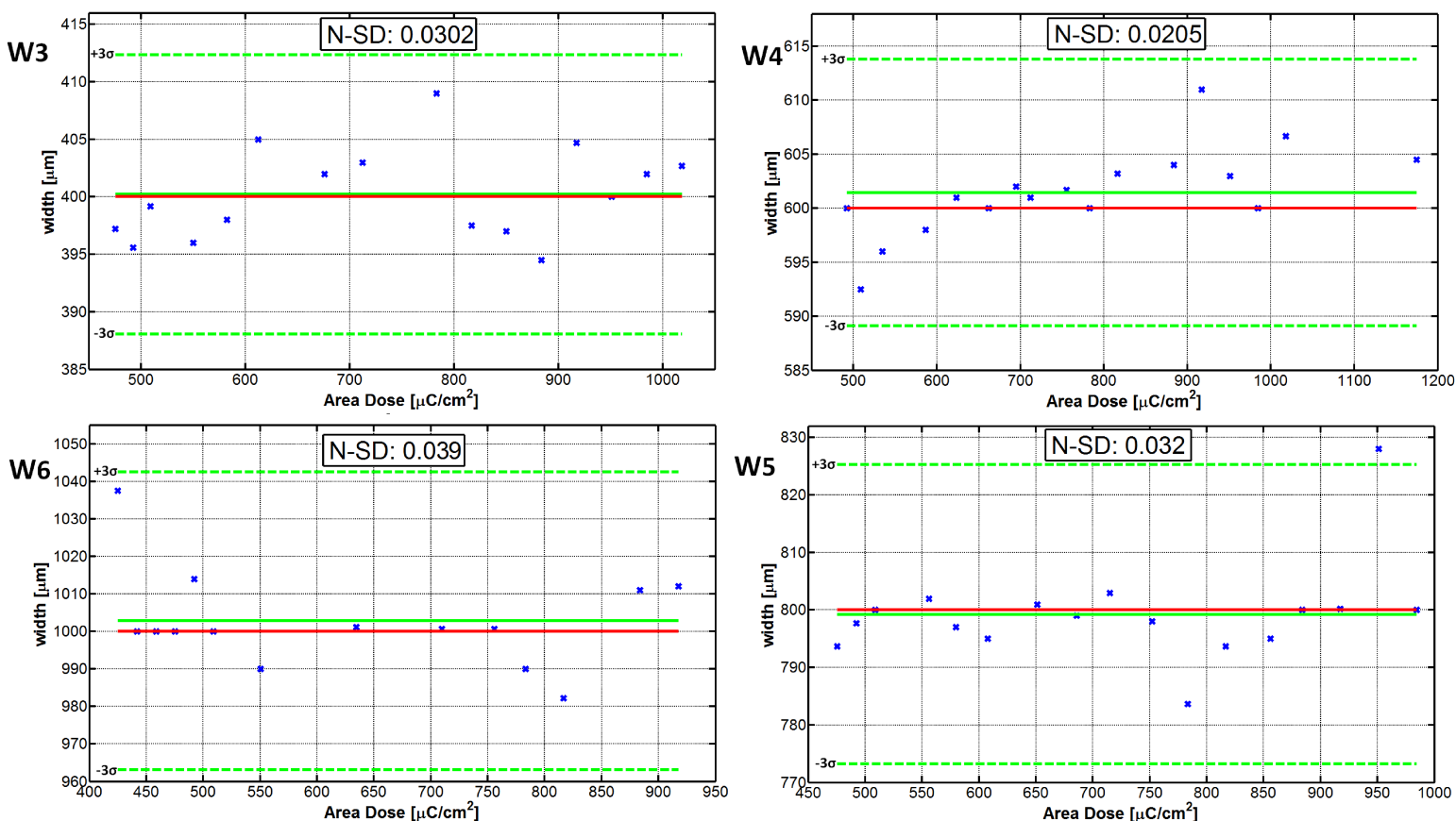

FIGURE 5. In the graph are reported all the experimental values of the nanosctructures' width (x) versus the Area Dose.

So, after these outcomes, we have performed a second writing cycle for investigating structures with higher thickness (Fig. 2b), with the aim of acquiring, also in this case, an high reproducibility. The patterning parameters are reported in Tab. 1. The image analysis for this new writing session and the N-SD value are represented in the four graphs in Fig. 5, whose composition has been described before. Its well evident that all the elements have values all under 5\%, demonstrating that these patternings are all reproducible in a wide dose range. We have also reported in Fig. 6, the SEM images for the four nanostructures obtained after the EBL process.
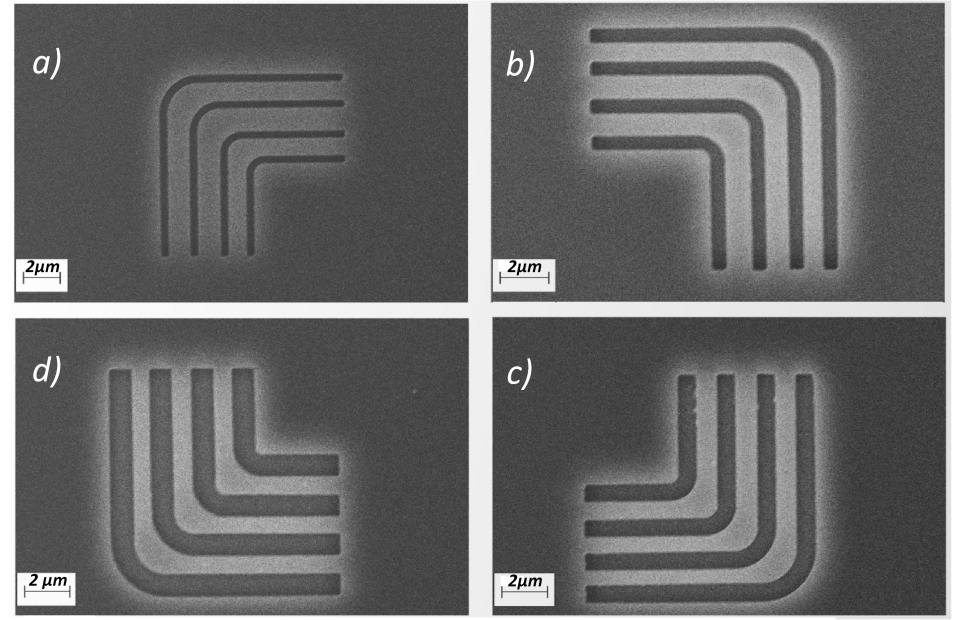

FIGURE 6. SEM images of the four nanostructure $(\mathrm{W} 1 \div \mathrm{W} 4)$ obtained by EBL with a developing time of $75 \mathrm{~s}$. The width of the L-shape structures increase in clockwise direction from a). 
Is results that the complex shapes of the CAD are well reproduced, as well as the borders are quite smooth. Besides, the presence of various sub-micron structures in a small area seems not to create any problem due to the proximity effect. This fact gives a further confirm the good quality of the process.

In electron beam lithography, together with exposure dose, one of the most influential parameter is the development time. In fact, Mohammad et al. [29] reports in their work that this parameter can affects the sensitivity of the e-resist, the exposure window and, above all, the ultimate resolution of the e-resist. For this reason, it has been necessary to investigate the same ADr with different development times, respectively, $40 \mathrm{~s}$ and $150 \mathrm{~s}$. Looking at the graph in Figure 7, it can be observed how dramatically development time influences the reproducibility of the lithography. In fact, a lower time respect the $75 \mathrm{~s}$ could create underexposed resist with irregular shapes. Instead, developing with higher times, the final width can be seriously modified by the solvent, because it could attack also the unexposed areas [29].

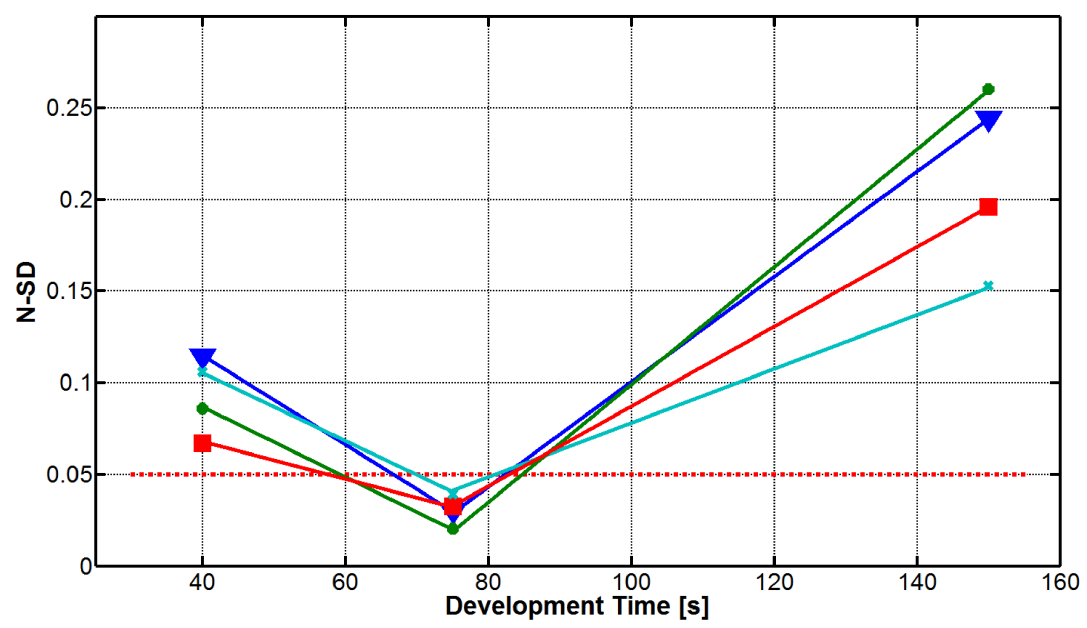

FIGURE 7. The values of the normalization standard deviation (N-SD) compared with the developing time for W1 ( $)$ ), W2 (•), W3 (口) and W4 (x).

\section{CONCLUSION}

A deeply investigation on the writing properties for a sub-micron resolution with high aspect ratio of the PMMA AR-P 672.08 is presented in this work. Through a statistical descriptive computing performed on the SEM images of the written patterning, it has been possible to found out the optimized Area Dose range for the a repeatable lithography process (N-SD values $<5 \%$ ) of sub-micrometer structures. The most important result regards the formation of 400 $\mathrm{nm}$ width structures in PMMA of $1.6 \mathrm{~m}$ thickness, using $75 \mathrm{~s}$ as developing time. In fact, we have obtained a very low N-SD value (3.03\%) and together with an high aspect ratio (4:1). Besides, it has been also investigated the effect of the developing time on the EBL process. We have found out that lower times lead to unexposed patterning with undefined shapes, while higher times can create uncontrolled increase of the width. This result gives us an additional confirmation that the developing time of $75 \mathrm{~s}$ is an optimized parameter. Besides, this study can open the way to develop an high performance use of the PMMA in single layer fabrication processes.

\section{ACKNOWLEDGMENTS}

The authors would like to thank Prof. Concita Sibilia from Department of Basic and Applied Sciences for Engineering (BASE), Sapienza University of Rome, for her support to research. 


\section{REFERENCES}

[1] W. Hu, K.Sarveswaran, and M. Lieberman, Vac. Sci. Technol. B 22, p. 1711 (2004).

[2] P. Hoffmann, I. Utke, A. Perentes, T. Bret, C. Santschi, and V. Apostolopoulos, "Comparison of fabrication methods of sub-100nm nano-optical structures and devices," in Nanophotonic Materials and Systems II, Proceedings SPIE 5925, edited by Z. Gaburro and S. Cabrini (SPIE, 2005) p. 592506.

[3] I. Maximov, E.-L. Sarwe, M. Beck, K. Deppert, M. Graczyk, M. Magnusson, and L. Montelius, Microelectronic Eng. 61, 449-454 (2002).

[4] N. Dutta, I. O. Mirza, and D. W. P. S. Sh and, Materials 3, 5283-5292 (2010).

[5] P. Dumon, W. Bogaerts, V. Wiaux, J. Wouters, S. Beckx, J. V. Campenhout, D. Taillaert, B. Luyssaert, P. Bienstman, D. V. Thourhout, and R. Baets, Phot. Tech. Lett. IEEE 16, 1328 - 1330 (2004).

[6] X. Zhao, Y.Xia, and G. Whitesides, J. Mater. Chem. 7, 1069 - 1074 (1997).

[7] Y. Li, X. Zhang, D. Wang, F. He, C. Ni, and L. Chi, J. Colloid \& Interface Sc. 458, 300-304 (2015).

[8] A. Boltasseva and V. M. Shalaev, Metamaterials 2, 1-17 (2008).

[9] O. Volciuc, T. Braniste, V. Sergentu, V. Ursaki, I. M. Tiginyanu, and J. Gutowski, "Fabrication of photonic crystal circuits based on gan ultrathin membranes by maskless lithography," in Nanotechnology VII, Proceedings SPIE 9519, edited by I. Tiginyanu (SPIE, 2015) p. 951904.

[10] E. Miele, G. Messina, M. Dipalo, V. Shalabaeva, A. Jacassi, S. Panaro, M. Malerba, P. Zilio, and F. D. Angelis, Microelectronic Eng. 141, 51-55 (2015).

[11] W. Smith and J. Hashemi, in Foundations Of Materials Science and Engineering (4. ed) (McGraw Hill, 2006) p. 509.

[12] G.R.Brewer, in Electron-Beam Technology in Microelectronic Fabrication (McGraw Hill, 1980).

[13] Y. Chen, Microelectronic Eng. 135, 57-72 (2015).

[14] H. Kollmann, M. X. Piao, S. F. Becker, D. Hou, C. Huynh, L. Kautschorand, G. Bosker, H. Vieker, A. Beyer, A. Glzhuser, N. Park, R. Vogelgesang, M. Silies, and C. Lienau, Nano Lett. 14, 4778-4784 (2014).

[15] B. Taylor, P. Michaux, A. S. M. Mohsin, and J. W. M. Chon, Opt. Express 22, 13234-13243 (2014).

[16] F. Domenici, C. Fasolato, E. Mazzi, L. D. Angelis, F. Brasili, F. Mura, P. Postorino, and F. Bordi, Colloids and Surfaces A: Physicochemical and Engineering Aspects 498, 168-175 (2016).

[17] S.Youn, T. Noguchi, M. Takahashi, and R. Maeda, Microelectronic Eng. 85, 918-921 (2008).

[18] V. Nock and R. J. Blaikie, Microelectronic Eng. 85, 1077-1082 (2008).

[19] C.J.Bettinger, R. Langer, and J. Borenstein, Angew. Chem. Int. Ed. 48, 5406-6415 (2009).

[20] C. M. Kolodziej and H. D. Maynard, Chem. Mater. 24, 774-780 (2012).

[21] P. Carlberg, M. Graczyk, E.-L. Sarwe, I. Maximov, M. Beck, and L. Montelius, Microelectronic Eng. 67/68, 203-207 (2003).

[22] D. Tu, M. Liu, L. Shang, C. Xie, and X. Zhu, "A zep520-lor bilayer resist lift-off process by e-beam lithography for nanometer pattern transfer," in IEEE Nano 2007 (IEEE, 2007), pp. 624-627.

[23] R. E. Howard, E. Hu, and L. D. Jackel, "A zep520-lor bilayer resist lift-off process by e-beam lithography for nanometer pattern transfer," in IEEE Transactions On Electron Devices (IEEE, 1981) p. 1378.

[24] A.Gangnaik, Y. M. Georgiev, B. McCarthy, N. Petkov, V. Djara, and J. D. Holmes, Microelectronic Eng. 123, 126-130 (2014).

[25] S. Yasin, D. G. Hasko, and H. Ahmeda, Appl. Phys. Lett. 78, p. 2760 (2001).

[26] S. P. Beaumont, P. G. Bower, T. Tamamura, and C. D. W. Wilkinson, Appl. Phys. Lett 38, p. 436 (1981).

[27] S. Cabrini and S. Hawata, in Nanofabrication Handbook (CRC Press, 2012).

[28] S. Institute Inc., JMP11 Quality \& Process Methods (SAS Institute Inc., Cary, NC, USA, 2014).

[29] M. A. Mohammad, M. Muhammad, S. K. Dew, and M. Stepanova, Nanofabrication Handbook (CRC Press, 2012), pp. 11-41. 